\title{
29. SOME RADIOLARIAN SAMPLES FROM THE WESTERN INDIAN OCEAN, DSDP LEG 25
}

\author{
Annika Sanfilippo and William R. Riedel, Scripps Institution of Oceanography, \\ La Jolla, California
}

\section{INTRODUCTION}

There was no radiolarian specialist in the shipboard scientific party on Leg 25 . Therefore, this note reports on only a few samples taken by shipboard paleontologists, specializing in other groups of microfossils, from cores for which additional biostratigraphic interpretation was considered desirable.

The localities represented, and the approximate depths of the cores investigated, are:

Site 239: $21^{\circ} 17.67^{\prime} \mathrm{S}, 51^{\circ} 40.73^{\prime} \mathrm{E} ; 4971 \mathrm{~m}$

Site 240: $03^{\circ} 29.24^{\prime} \mathrm{S}, 50^{\circ} 03.22^{\prime} \mathrm{E} ; 5082 \mathrm{~m}$

Site 248: $29^{\circ} 31.78^{\prime} \mathrm{S}, 37^{\circ} 28.48^{\prime} \mathrm{E} ; 4994 \mathrm{~m}$

Site 249: $29^{\circ} 56.99^{\prime} \mathrm{S}, 36^{\circ} 04.62^{\prime} \mathrm{E} ; 2088 \mathrm{~m}$

The concepts of species applied in this report conform to those used by Riedel and Sanfilippo (1971) and Sanfilippo and Riedel (1973).

\section{SITE 239}

Samples $8-1,89-91 \mathrm{~cm}$ and $8-3,85-86 \mathrm{~cm}$ contain few, moderately well preserved (somewhat dissolved) radiolarians of a variety of ages ranging from early Eocene to middle Miocene. Included are: collosphaerids, Cannartus (?) petterssoni, Ceratospyris articulata, Dictyospyris discus, Dorcadospyris dentata, Giraffospyris lata, Liriospyris stauropora, Tristylospyris triceros, Bekoma campechensis, Buryella clinata, Lychnocanoma anacolum, L. auxilla, Phormocyrtis cubensis, $P$. striata exquisita, $P$. striata striata, Stichocorys wolffii, Thyrsocyrtis hirsuta hirsuta, T. hirsuta tensa, T. rhizodon, T. triacantha, Podocyrtis fasciolata, $P$. mitra, $P$. papalis, $P$. sinuosa, and Theocampe mongolfieri.

Samples 9-3, 105-106 cm; 11, CC; 12, CC; and 15, CC contain no, or very rare, radiolarians; insufficient for interpretation.

\section{SITE 240}

Samples 1-3, 86-88 cm; 1-5, 132-134 cm; 1-6, 133-135 $\mathrm{cm}$; and 1, CC were examined. All contained abundant, well preserved Quaternary radiolarians including Spongaster tetras, Ommatartus tetrathalamus, Theocorythium trachel- ium, Pterocorys hertwigii, and Lithopera bacca, and no admixed Tertiary forms.

Sample 3-1, 104-106 cm contains no radiolarians.

\section{SITE 248}

Samples with radiolarians sufficient for interpretation are shown in Table 1. Others examined, in which radiolarians are absent or very rare, are $10-1,100-102 \mathrm{~cm}$; $13-6,132-134 \mathrm{~cm} ; 14-1,60-62 \mathrm{~cm} ; 14-4,90-92 \mathrm{~cm} ; 14-5$, $70-72 \mathrm{~cm} ; 14-6,70-72 \mathrm{~cm}$; and $14, \mathrm{CC}$. The radiolarian assemblages in Cores 10 to 12 seem to belong in the lower part of the Phormocyrtis striata striata Zone, which is approximately equivalent to the Hantkenina aragonensis Zone and the boundary between the Discoaster lodoensis and $D$. sublodoensis zones (late early Eocene).

\section{SITE 249}

Samples 23-3, 121-123 cm; 23-4, top; 23-5, 130-132 cm; 24-1, 106-108 cm; 26-1, 38-40 cm; 26-1, 121-123 cm; 26-2, $40-42 \mathrm{~cm} ; 27-2,80-82 \mathrm{~cm} ; 27-3,125-127 \mathrm{~cm} ; 28-1,78-79$ $\mathrm{cm} ; 29$, CC; 30-1, $128-129 \mathrm{~cm} ; 30-3,117-118 \mathrm{~cm} ; 30-4$, $18-19 \mathrm{~cm} ; 30$, CC; $31-2,44-45 \mathrm{~cm}$; and $31-3,135-136 \mathrm{~cm}$ contain few to common, poorly to moderately preserved radiolarians. They are absent or very rare in $23-5,89-91 \mathrm{~cm}$; $25-2,102 \mathrm{~cm} ; 25-3,48-50 \mathrm{~cm}$; and $32-1,104-106 \mathrm{~cm}$. The assemblages evaluated from Cores 23 to 26 appear to be early Late Cretaceous in age.

\section{REFERENCES}

Riedel, W. R. and Sanfilippo, A., 1971. Cenozoic Radiolaria from the western tropical Pacific, Leg 7. In Winterer, E. L. et al., Initial Reports of the Deep Sea Drilling Project, Volume 7: Washington (U.S. Government Printing Office), p. 1529-1672.

Sanfilippo, A. and Riedel, W. R., 1973. Cenozoic Radiolaria (exclusive of Theoperids, Artostrobiids and Amphiphydacids) from the Gulf of Mexico, Deep Sea Drilling Project, Leg 10. In Worzel, J. L., Bryant, W., et al., Initial Reports of the Deep Sea Drilling Project, Volume 10: Washington (U. S. Government Printing Office), p. 475-612. 
TABLE 1

Radiolarians at Site 248

\begin{tabular}{|c|c|c|c|c|c|c|c|c|c|c|}
\hline 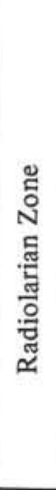 & Samples & Species & & 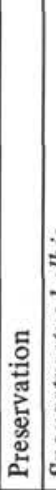 & 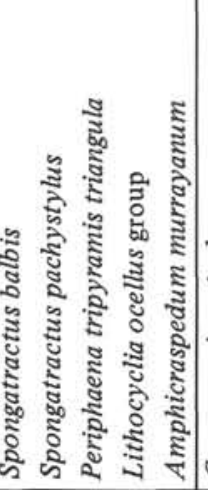 & 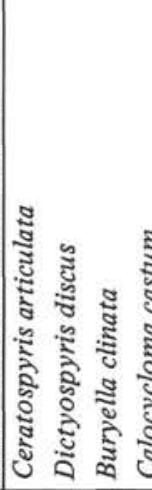 & 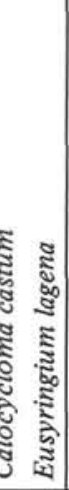 & 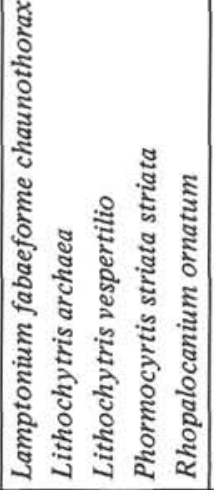 & 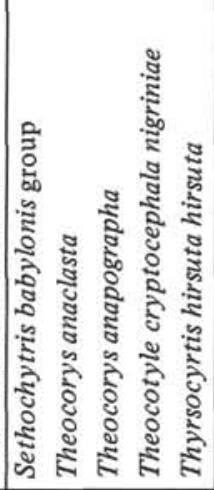 & 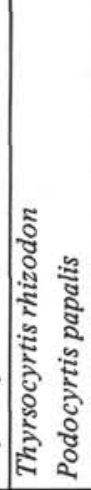 \\
\hline \multirow{2}{*}{ 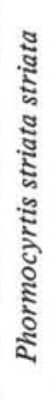 } & $\begin{array}{l}248-10-2, \\
248-10, \text { CC } \\
248-11-2, \\
248-11-3, \\
248-12-1, \\
\end{array}$ & $\begin{array}{l}147-149 \\
70-72 \\
137-138 \\
80-82 \\
\end{array}$ & $\begin{array}{l}\mathrm{F} \\
\mathrm{F} \\
\mathrm{F} \\
\mathrm{F} \\
\mathrm{C}\end{array}$ & \begin{tabular}{l|}
$\mathrm{M}$ \\
$\mathrm{M}$ \\
$\mathrm{P}$ \\
$\mathrm{M}$ \\
$\mathrm{M}$
\end{tabular} & & \multicolumn{2}{|l|}{$++R$} & & & \\
\hline & $\begin{array}{l}248-12-3, \\
248-12-4, \\
248-12-6, \\
248-12-6, \\
248-12-6, \\
248-12, \text { CC }\end{array}$ & $\begin{array}{l}80-82 \\
30-32 \\
50-52 \\
60-62 \\
120-122\end{array}$ & $\begin{array}{l}\mathrm{C} \\
\mathrm{C} \\
\mathrm{C} \\
\mathrm{C} \\
\mathrm{F} \\
\mathrm{C}\end{array}$ & \begin{tabular}{l|l}
$\mathrm{M}$ & \\
$\mathrm{M}$ & \\
$\mathrm{M}$ & \\
$\mathrm{M}$ & $\mathrm{M}$ \\
$\mathrm{M}$ &
\end{tabular} & $\begin{array}{r}+ \\
\mathrm{R}^{+} \\
\end{array}$ & $\begin{array}{l}\mathrm{R} \\
\mathrm{R} \\
\mathrm{R} \\
\mathrm{F}\end{array}$ & $\mathrm{R}$ & 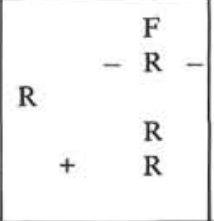 & $\ldots-{ }^{\mathrm{R}}$ & - \\
\hline
\end{tabular}





\section{PLATE 1}

Cretaceous Radiolarians from Site 249

(Magnification 255X)

Figures 1-17 Assemblage from 249-24-1, 106-108 cm; S1.1.

Figures 1, 2 Spongodiscids gen. et sp. indet.

1. $\mathrm{P} 31 / 2$.

2. $\mathrm{X} 31 / 1$.

Figures 3, $4 \quad$ Hemicryptocapsa sp.

3. $\mathrm{R} 36 / 3$.

4. B21/1.

Figure $5 \quad$ Amphipyndax? sp.; W41/3.

Figure 6. Lithomitra pseudopinguis Tan Sin Hok; N33/4.

Figure $7 \quad$ Theocorys sp.; G45/0.

Figure $8 \quad$ Theocorys? sp.; W41/0.

Figures 9, $10 \quad$ Stichocapsa sp.

9. D24/4.

10. $\mathrm{S} 27 / 0$

Figure $11 \quad$ Eucyrtis sp.; N26/0.

Figure 12 Dictyomitra sp.; R45/3.

Figure $13 \quad$ Eucyrtidium sp.; N34/0.

Figure $14 \quad$ Lithomitra sp.; P31/0.

Figure 15 Amphipyndax mediocris Tan Sin Hok; 021/0.

Figure 16 Amphipyndax? sp.; V33/2.

Figure $17 \quad$ Lophophaena sp.; R22/0.

Figures 18-22 Assemblage from 249-26-2, 40-42 cm; S1.1.

Figure $18 \quad$ Hagiastrid; N51/4.

Figure $19 \quad$ Tricolocapsa sp.; S14/4.

Figure $20 \quad$ Cyrtocapsa; V42/2.

Figure $21 \quad$ ?Eucyrtis sp.; C45/0.

Figure $22 \quad$ Dictyomitra sp.; J47/4. 


\section{PLATE 1}

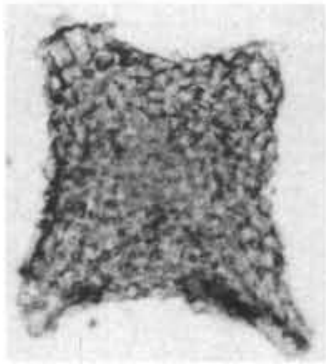

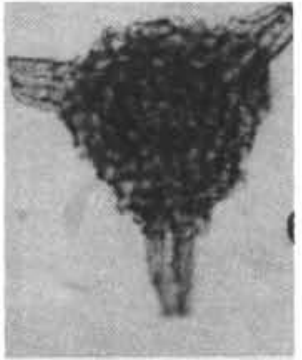

2

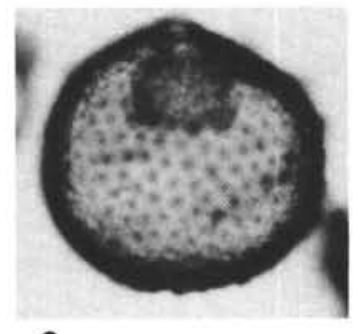

3
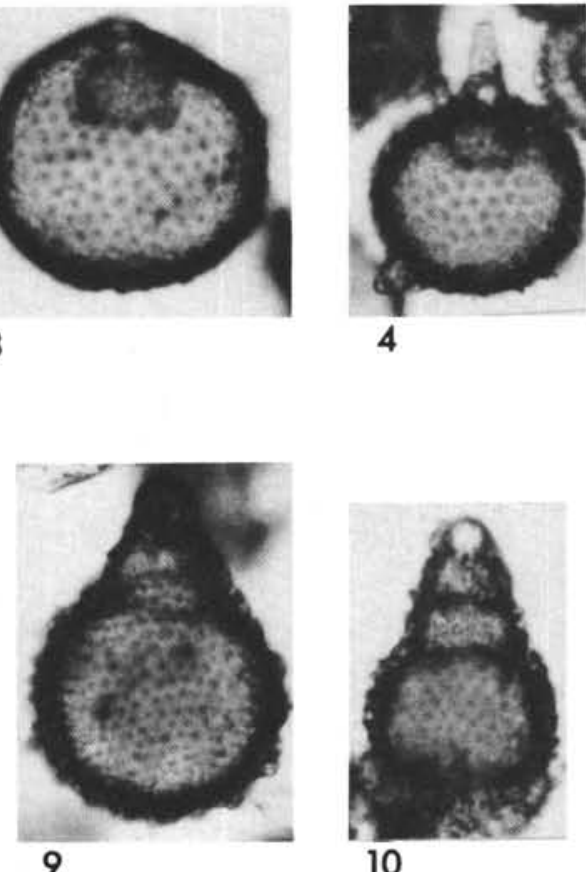

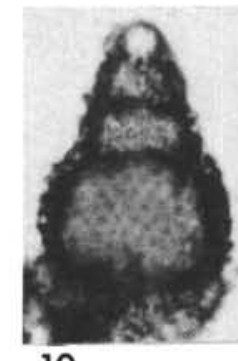

10

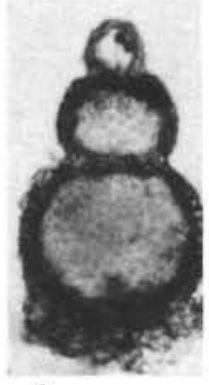

6
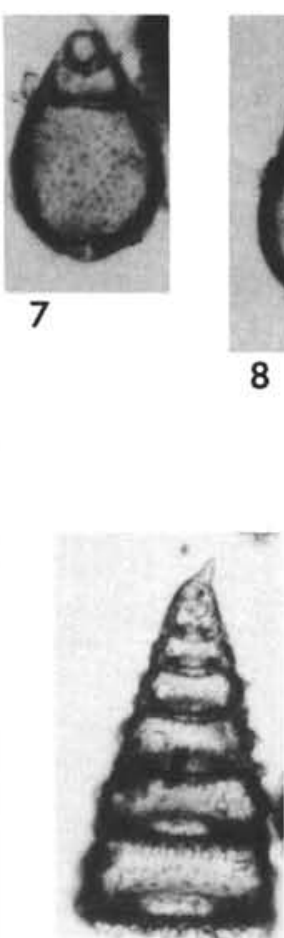

13

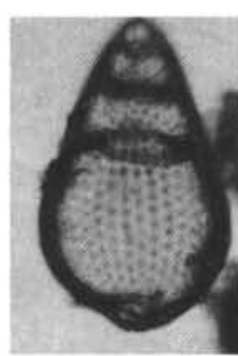

8

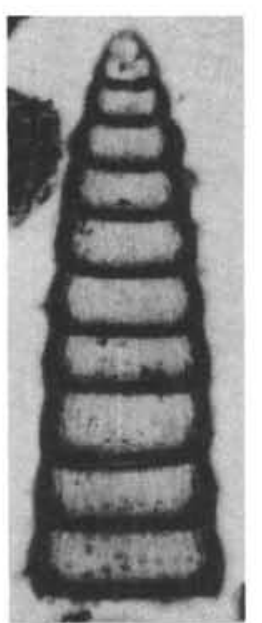

12

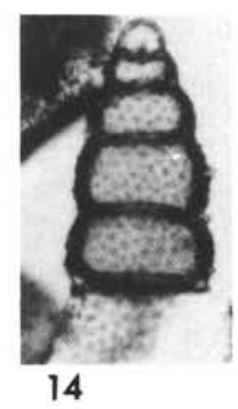

14
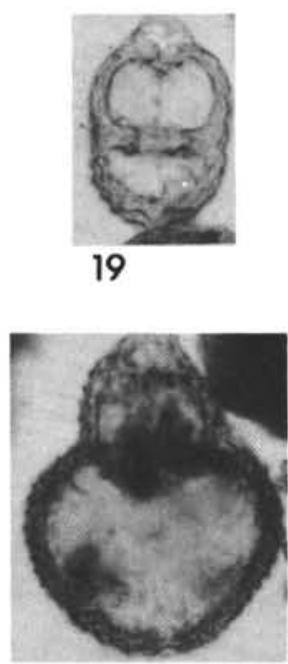

20
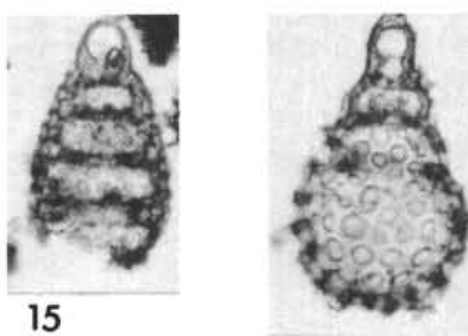

16

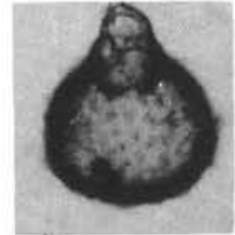

5

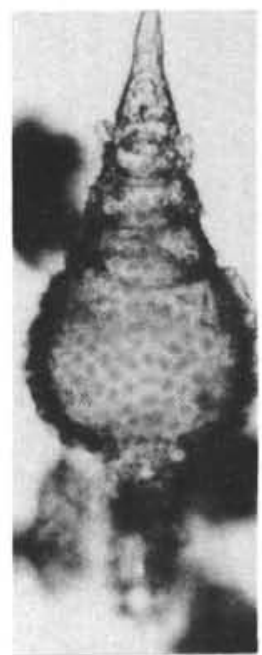

11

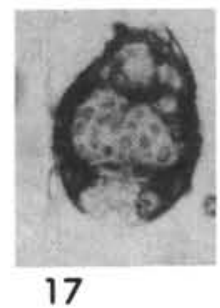

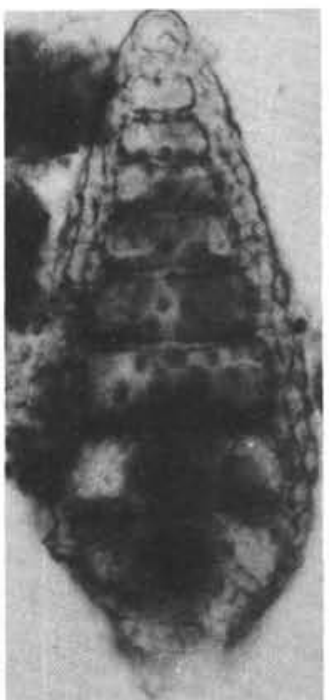

21

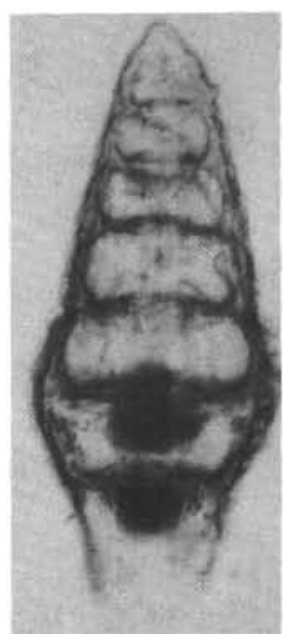

22 